\title{
The Diagnostics of Fetal Heart Defects in the First and Early Second Trimester - Early Fetal Echocardiography
}

\author{
Die Diagnostik fetaler Herzfehler im 1. und frühen 2. Trimenon - \\ frühe fetale Echokardiografie
}

Authors

Affiliation
U. Gembruch, A. Kempe, A. Hellmund, B. Rösing, A. Willruth, C. Berg, A. Geipel

Department of Obstetrics and Prenatal Medicine, University Hospital, Bonn, Germany

\author{
Key words \\ - ultrasound \\ - congenital heart defects \\ - fetal echocardiography \\ - first trimester \\ prenatal diagnosis \\ - fetus

\section{Schlüsselwörter \\ - Ultraschall \\ - angeborene Herzfehler \\ - fetale Echokardiografie \\ - erstes Trimester \\ - pränatale Diagnostik \\ - Fet}

\section{Deutschsprachige} Zusatzinformationen online abrufbar unter: www.thieme-connect.de/ ejournals/toc/gebfra

$\begin{array}{ll}\text { received } & 30.10 .2013 \\ \text { revised } & 26.11 .2013 \\ \text { accepted } & 26.11 .2013\end{array}$

Bibliography

Dol http://dx.doi.org/ 10.1055/s-0033-1360194

Geburtsh Frauenheilk 2013; 73 : 1209-1217 (c) Georg Thieme Verlag KG Stuttgart · New York ISSN 0016-5751

\section{Correspondence}

\section{Prof. Ulrich Gembruch}

Department of Obstetrics and Prenatal Medicine,

University Hospital Bonn

Sigmund-Freud-Straße 25

53105 Bonn

ulrich.gembruch@

ukb.uni-bonn.de

\section{Abstract \\ $\nabla$}

Examinations of the fetal heart are increasingly being conducted as part of first-trimester screening, either as a sole visualisation of the fourchamber view or a visualisation of the fourchamber view and both ventricular outflow tracts or as a segmental approach as part of complete fetal echocardiography. Alongside anamnestic risks, markers for heart defects that become apparent during a first-trimester screening, such as thickened nuchal translucency, an abnormal blood flow velocity profile in the ductus venosus and the presence of tricuspid valve regurgitation, are indications for an early echocardiogram in this high-risk group. However, heart defects most often occur in fetuses in a low-risk group who display none of the markers mentioned. An increasing number of examiners are therefore also conducting a fetal echocardiogram as part of the detailed first-trimester screening of fetal organs. Up to $80 \%$ of severe heart defects can be diagnosed as early as the 12th and 13th weeks of gestation. Apart from the examiner's experience and the best possible equipment, prerequisites are a consistent visualisation of all cross-sections and connections following a strict protocol, including transvaginal approach in some cases, and, in very rare cases, during a repeat examination a few days later. Nevertheless, primarily due to the intrauterine development of some heart defects, a further echocardiographic examination should always be carried out in the second trimester, when the detection rate is up to $10 \%$ higher.

\section{Zusammenfassung \\ $\nabla$}

Im Rahmen der Ersttrimesteruntersuchung erfolgt zunehmend auch die Untersuchung des fetalen Herzens entweder als alleinige Darstellung des Vierkammerblicks oder des Vierkammerblicks und der beiden ventrikulären Ausflusstrakte oder als segmentales Vorgehen im Sinne einer kompletten fetalen Echokardiografie. Neben einem anamnestischen Risiko sind Hinweiszeichen für Herzfehler, die während der Ersttrimesteruntersuchung auffallen, wie eine verdickte Nackentransparenz, ein abnormales Blutflussgeschwindigkeitsprofil im Ductus venosus und das Vorliegen einer Trikuspidalklappenregurgitation, Indikationen zu einer frühen Echokardiografie in diesem Hochrisikokollektiv. Andererseits treten die meisten Herzfehler bei Feten auf, die einem Niedrigrisikokollektiv angehören und keine der genannten Hinweiszeichen bieten. Daher gehen immer mehr Untersucher dazu über, im Rahmen der detaillierten Ersttrimesteruntersuchung der fetalen Organe auch eine fetale Echokardiografie durchzuführen. Bis zu $80 \%$ der als schwer einzustufenden Herzfehler können so bereits mit 12 und 13 SSW diagnostiziert werden. Voraussetzung sind aber neben der Erfahrung des Untersuchers und einer optimalen Geräteausstattung die konsequente, einem festen Protokoll folgende Einstellung aller Schnittebenen bzw. Konnektionen, auch unter Einsatz der transvaginalen Echokardiografie und in sehr seltenen Fällen auch durch eine erneute Untersuchung wenige Tage später. Trotzdem - dies ist hauptsächlich der intrauterinen Entwicklung einiger Herzfehler geschuldet - sollte immer eine weitere echokardiografische Untersuchung im 2. Trimenon erfolgen, deren Entdeckungsrate bis zu 10\% höher ist. 


\section{Introduction}

The sonographic first-trimester screening established during the last decades for fetal aneuploidies through measuring nuchal translucency and the associated higher demands on examiners and equipment, particularly in conducting an advanced first-trimester screening including the examination of the nasal bone and the blood flow pattern into the ductus venosus and through the tricuspid valve, have resulted in increasingly more pregnant women undergoing this examination between the $12 \mathrm{th}+0$ and 13 th +6 weeks of pregnancy on the one hand, and on the other, to an increasing number of examiners dealing with the presentation of fetal anatomy in this stage of pregnancy and trying to diagnose as many non-chromosomal malformations as possible as part of this examination.

Even if the first-trimester screening is initially defined via the risk evaluation for aneuploidies and particularly through the potential reduction of the invasive interventions associated with the risk of miscarriage, and early diagnostics to exclude malformations has only been carried out for fetuses with thicker nuchal translucency, it still offers the possibilities for all fetuses to be examined, even those with normal nuchal translucency thickness. In many clinics, the sonographic first-trimester screening is now a comprehensive, detailed depiction of the fetal anatomy and does not merely serve the purpose of accurately determining the gestational age, detecting a multiple pregnancy and determining the chorionicity as well as screening for fetal aneuploidies, but rather is performed with the goal of detecting severe fetal anomalies early and potentially weeks before the second-trimester screening, independent of maternal age, anamnestic risk or the thickness of nuchal translucency $[1,2]$.

Similarly to the advanced organ diagnostics and the fetal echocardiogram in the second trimester, the sensitivity and specificity of the first-trimester screening is dependent on the experience of the examiner, the precise representation of the fetal organs in accordance with a protocol, as well as the quality of the ultrasound equipment and often a transvaginal sonogram being carried out in addition to the transabdominal one. This applies in particular when examining the heart of a fetus. The attentiveness of the examiner or the desire for a complete sampling of the fetal organs influence the detection rate of non-chromosomal malformations, which is higher for the same malformations in the group of fetuses with thicker nuchal translucency than in the group with normal nuchal translucency thickness [3].

The prevalence of congenital heart defects (CHD) is 10 out of 1000 live births, whereby 3-4/1000 babies born alive displayed a major CHD that required cardiological interventions and/or cardiosurgery. Approximately one-third of children with congenital malformations have a heart defect and, despite all the advances made in their treatment, heart defects remain the most significant cause of mortality and morbidity among infants and children. In the last 30 years, as a result of the inclusion of the systematic examination of the four-chamber view and partly also both ventricular outflow tracts in the ultrasound screening examination between the 18th and 24th weeks of pregnancy and the increasingly frequent use of colour Doppler devices, coupled with the intensive training of examiners, a detection rate of 60$80 \%$ for major CHDs as well as minor CHD, such as small ventricular septal defects, was achieved in the second trimester. This is advantageous for many of these children, as the knowledge of the existence of a major heart defect enables the precise planning of perinatal treatment in advance. In particularly, those defects benefit considerably from prenatal diagnosis for which a sufficient perfusion of the pulmonary or circulatory system is dependent on the ductus arteriosus and/or the foramen ovale remaining open. Both pre-operative morbidity as well as mortality can be significantly reduced for some major heart defects, as this has been well established for the transposition of the large arteries, the hypoplastic left heart and the coarctatio aortae (aortic coarctation) [4]. Furthermore, it appears that, in some situations, fetuses with severe outflow tract anomalies benefit from interventions controlled by intrauterine ultrasound (balloon dilatation of a semilunar valve; opening of a closed or highly restrictive foramen ovale), thereby preventing the rapid development towards the hypoplastic ventricle during pregnancy and so the possibility of a univentricular repair can be reduced or intervention can have a life-saving effect in cases of hydrops [5].

\section{Features of Early Fetal Echocardiography} $\nabla$

Although it has been used successfully since the early 1990s [68], early fetal echocardiography has only been set up at the relevant centres within the last 10 years and is now used worldwide for risk groups between the 12th and 14th weeks of pregnancy. The huge improvement in ultrasound technology allows this examination to be carried out transabdominally for the majority of fetuses. The global establishment of aneuploidy screenings with the measurement of nuchal translucency (NT measurement) and their expansion to further markers, such as nasal bone, blood flow velocity profile of the ductus venosus (DV) and tricuspid valve regurgitation (TR) has been crucial. It is increasingly being complemented by a comprehensive first-trimester screening of the fetus and its organs, whereby the four-chamber view and, with increasing frequency, both ventricular outflow tracts are already part of the examination protocol. An increasing number of examiners now have sufficient experience with detailed first-trimester screening, including the visualisation of the most important cardiac cross-sections through two-dimensional and colourcoded echocardiography. A further reason for the current rapid spread of detailed early fetal echocardiography is that fetuses with thickened nuchal translucency have an abnormal flow profile in the ductus venosus and, in the presence of a tricuspid valve regurgitation, have increased risk of the presence of a heart defect and the exclusion of which should, in the interests of the parents, if possible take place immediately on detection of one of these abnormalities, and not 8 weeks later in the second trimester. As the majority of fetuses with heart defects have neither a genetic risk nor one of the abnormalities mentioned in the firsttrimester screening, some call for a fetal echocardiography to be carried out as standard, as part of the detailed first-trimester screening $[1,9]$.

As this also applies generally to the diagnosis of malformations and to the echocardiogram in particular, their detection rate is dependent on a variety of factors, both in the second trimester, but even more so in the first trimester, due to the extreme difficulty of carrying out a comprehensive examination of the fetal heart $[4,10,11]$ :

1. Time of examination (10th-11th week of pregnancy, 12th13th week of pregnancy, 14th - 15th week of pregnancy) and respective visualisation of the cardiac structures [12-14]; 
2. Scope of examination - screening by markers only (NT, DV, TR), through the visualisation of the four-chamber view, through the visualisation of the four-chamber view and outflows of both great arteries, or through complete early echocardiography with segmental procedure $[1,2,4,11,13,16,17]$;

3. Use of colour Doppler [4];

4. Equipment quality and use of high frequency transducers (transabdominal and transvaginal) [18];

5. Consistent use of transvaginal echocardiography, in particular in the case of a non-optimal depiction of cardiac structures [7, $8,12-14,19]$;

6. Attentiveness of the examiner: higher detection rates for the same fetal anomalies in groups with noticeable markers (NT, DV, TR) [3];

7. Detection rates for a major CHD vs. a minor CHD and the inconsistent definition of a major CHD vs. a minor CHD [20].

The difficulties of an early examination of the fetal heart have been documented in many studies. Alongside the malformations of the brain and kidneys that are not yet manifest in the first trimester and therefore not visible in a first-trimester examination, heart defects are also one of the severe anomalies to have very low detection rates in all studies without exception - including in the second trimester - due to the particular difficulties experienced when examining this organ. A meta-analysis summarised 36237 pregnancies that were examined over eight centres from 1993 to 2008 between the 11th and 13th week of pregnancy. The average detection rate for major CHD was 29\% (95\% CI: 25-33\%); the pooled detection rate for major CHD between the 11th and 13th week of pregnancy was only $17 \%$ (95\% CI: 10-25\%) [21]. Very poor results were also shown by a large Swedish study which investigated and compared the efficiency of diagnostics to exclude malformations in the first and second trimesters without a strict investigation protocol between 1999 and 2002 [22]. The detection rate for heart defects was particularly bad in both groups, whereby only the four-chamber view was to be depicted in both groups; an inadequate image of the four-chamber view was accepted in the early group, insofar as the nuchal translucency was normal; a fetal echocardiogram was only conducted for fetuses with a nuchal translucency over $3.5 \mathrm{~mm}$. The poor detection rates for major heart defects were not significantly different in each group and amounted to $11 \%(7 / 61)$ and $15 \%(9 / 60)$ respectively; finally, the heart of only one fetus was recognised as abnormal in the early examination as a result of dextrocardia, and for three other fetuses, the severe heart defect was first detected in the second ( $5 \%$ before 22 nd week of pregnancy) or third trimester [22].

Considerably higher detection rates for non-chromosomal anomalies were found in sonographic first-trimester screenings that followed strict investigation guidelines, which were carried out at centres and by examiners that already have a lot of experience in first-trimester screening as part of aneuploidy screening. Many more malformations were able to be diagnosed for both time intervals in a prospective cohort study at two large British centres with investigators certified by the Fetal Medicine Foundation U.K. for both the first- and second-trimester ultrasound examinations [23]. After excluding fetuses with aneuploidy (0.7\%), 488/44,859 (1.1\%) of euploid fetuses had a severe anomaly, 213 (43.6\%) of which were diagnosed as early as the first-trimester screening between the 11th and 13th weeks of pregnancy; anomalies were detected in a further 262 out of 42,643 (262/488, 53.7\%) fetuses between the 14th and 18th weeks of pregnancy. Certain anomalies, such as acrania/anencephaly, alo- bar holoprosencephaly, abdominal wall defects, body-stalk anomalies and megacystis in the case of urethral obstruction were already recognised in the first-trimester screening, while others, such as heart defects, were detected only to a small degree (the four-chamber view had to be presented in this study in the first trimester). Detection was dependent on whether they were accompanied by thicker nuchal translucency (34\% of major heart defects) (detection rate were about $50 \%$ for hypoplastic left heart and double outlet right ventricle and about 30\% for transposition of the major arteries, atrioventricular septal defects, coarctatio aortae) [23].

\section{Cross-Sections, Approaches and the Time of Early Fetal Echocardiography \\ $\nabla$}

As in the second trimester, it is not only the four-chamber view and ventricular outflow tracts that are examined two-dimensionally and using colour Doppler in the fetal echocardiogram, but rather a segmental approach is used, whereby, after determining the location and the subsequent determination of the left and right-hand sides of the fetal body starting from the visceral situs, the visceral arterial, atrioventricular and ventriculoarterial connections are presented two-dimensionally as well as in the form of a colour Doppler coded echocardiogram [24,25]. The abdominal situs, location of the heart, four-chamber view, right and left outflow tract, crossing over of the major arteries and threevessel trachea view are depicted by means of a two-dimensional and colour Doppler echocardiogram; the confluence of one or two pulmonary veins in the left atrium can be depicted; the presence of a right aortic arch, an arteria lusoria and a persisting left superior vena cava are to be watched out for. The approach completely corresponds to that which is called for in the echocardiographic examination in the second trimester [26,27].

The depiction of the heart in the B-scan as well as by colour Doppler is greatly limited in the first trimester due to its small size. The growth of the fetal heart between the 10th, 13th and 15 th week of pregnancy is considerable $[12,28]$; the transverse total diameter at the level of the atrioventricular valves increases from $4.4 \mathrm{~mm}$ in the 10 th week of pregnancy to $11.4 \mathrm{~mm}$ in the 15th week of pregnancy, and the surface of the heart increases from $21 \mathrm{~mm}^{2}$ to $140 \mathrm{~mm}^{2}$ over the same period [12], which, given the small size of the relevant structures, significantly improves their visualisation week by week - the end-diastolic ventricle diameter is $1.0 \mathrm{~mm}$ in the 10th week of pregnancy and $3.0 \mathrm{~mm}$ in the 15th week of pregnancy, the diameter of the aorta and truncus pulmonalis is between $0.6 \mathrm{~mm}$ and $0.7 \mathrm{~mm}$ in the 10 th week of pregnancy and between $2.0 \mathrm{~mm}$ and $2.2 \mathrm{~mm}$ in the 15 th week of pregnancy $[10,12,14,19]$. Newer studies show that by the 13 th week of pregnancy almost all relevant structures can be presented transabdominally, but a transvaginal echocardiogram must be performed at earlier gestational age in order to achieve this. The most difficult is the depiction of the pulmonary veins (only done using colour Doppler), which can be achieved in the 12th and 13th weeks of pregnancy with sufficient persistence on the part of the examiner and the most up-to-date equipment [10,12-14, $18,19,29]$. The optimum time for the early fetal echocardiogram is currently between the 12th and 15th weeks of pregnancy, whereas echocardiography is increasingly being carried out as part of the large first-trimester screening in the (12th and) 13th week of pregnancy, as, on the one hand, the algorithms for a risk evaluation regarding chromosomal anomalies are valid at this 
time and, on the other hand, the cardiac cross-sections can be adequately depicted in almost all cases by means of transabdominal sonography, meaning that an additional transvaginal examination is not required. In the 13th week of pregnancy, all cardiac structures can generally be visualised transabdominally in the segmental approach, and a transvaginal examination with a transducer or a delayed second examination is only necessary for very few fetuses.

The colour Doppler is of particular importance in the early echocardiogram, as it is frequently indispensable in locating vessels; the B-scan and colour Doppler complement each other, as the optimum angle for depicting a vessel in the B-scan is $90^{\circ}$, but is $0^{\circ}$ in the colour Doppler, and so, for example, when the fetus is in a particular position, the aorta can be depicted well in the B-scan, but the truncus pulmonalis can only be depicted using colour Doppler. Due to their small size, hypoplastic arteries are primarily only recognised by a colour Doppler echocardiogram if there is an obstruction of the semilunar valve. In the presence of abnormalities, advantage should always be attempted to be taken of the better resolution of a higher frequency transvaginal or transabdominal ultrasound $[6-8,10,12,13,18]$, which can considerably increase diagnostic accuracy in the depiction of smaller atrioventricular septal defects and ventricular septal defects, whether isolated or as part of a tetralogy of Fallot or a double outlet right ventricle. Examinations in the 14th and 15th weeks of pregnancy enable an even better visualisation of the cardiac structures and should be carried out to differentiate abnormal findings in an echocardiogram in the previous weeks or in the case of any other abnormalities that would indicate an echocardiogram $[4,24]$. The three-dimensional echocardiogram using STIC (spatio-temporal image correlation) technology can generate good volumes with high frequency vaginal transducers, but is inferior in the diagnosis and differentiation of heart defects in the first trimester due to its lower resolution in two-dimensional echocardiography [30]. In any case, discussion will continue in future as to whether, in the case of the complete replacement of the first-trimester aneuploidy screening with the analysis of cell-free fetal DNA in the mother's blood, it would be sensible to carry out the first-trimester screening and the echocardiogram between the 13th and 15th weeks of pregnancy, as some working groups have long been proposing $[8,31]$.

\section{Markers for the Presence of a Heart Defect \\ $\nabla$}

Heart defects are some of the anomalies that accompany transient markers during the late-early and early-second trimester, which are evaluated as part of the aneuploidy screening. Thickened nuchal translucency, abnormal blood flow pattern in the ductus venosus as well as the presence of a tricuspid valve regurgitation are markers not only for aneuploidies with and without heart defects, but also for heart defects in euploid fetuses. The risk of the existence of a heart defect in euploid fetuses increases exponentially with the thickness of nuchal translucency (NT). A meta-analysis of 10 studies with 192922 euploid fetuses with a thicker NT showed that the prevalence of a major CHD was $1.5 \%$ for a thickness of between $2.5 \mathrm{~mm}$ and $3.4 \mathrm{~mm}$ (95th - 99th percentiles), $3.3 \%$ in the case of a NT of $3.5-4.4 \mathrm{~mm}, 5.5 \%$ for a NT of 4.5-5.4 mm, 15\% for an NT of 5.5-6.6 mm and 19\% for an NT between $6.5 \mathrm{~mm}$ and $8.4 \mathrm{~mm}$ [32]. In comparison with a normal prevalence of $8 / 1000$ live births, the risk of a heart defect in the $2.5 \mathrm{~mm}$ to $3.4 \mathrm{~mm}$ nuchal thickness group increases 2.5 -fold, with a 10-fold increase in risk in the group with an NT above $3.5 \mathrm{~mm}$ [32]. The individual heart defects do not appear to differ with regard to nuchal thickness [33].

In any case, nuchal thickness, the blood flow velocity profile in the ductus venosus and the presence of a tricuspid valve regurgitation are not independent markers, as they are all influenced by changes in haemodynamics. Slight haemodynamic changes in the case of heart defects and other illnesses and a delayed development of myocardial diastolic and systolic function are primarily manifested in the first trimester, as diastolic function, in particular the compliance of the heart, and systolic function are still low and, due to the high placental resistance, the cardiac afterload is still high. In the second trimester, in the case of considerably better diastolic and systolic cardiac function and considerably lower cardiac afterload, the higher pulsatility of the blood flow velocity profile of the ductus venosus only occurs in the case of severe right heart obstruction [34]. The thickness of the nuchal translucency, pulsatility in the blood flow pattern of the ductus venosus and the presence of a tricuspid valve regurgitation therefore correlate with one another [15,35-39]. Abnormal blood flow patterns were found in $20-40 \%$ of fetuses with thicker nuchal translucency, while their prevalence is only $2-3 \%$ in fetuses with normal nuchal translucency thickness $[35,38,40]$. Furthermore, the thickness of the nuchal translucency correlates with the frequency of an abnormal blood flow pattern in the ductus venosus and a tricuspid valve regurgitation. All three markers for heart defects are also only transiently visible at the time of the first-trimester screening.

The connection between these markers and the presence of a heart defect implies that a thicker nuchal translucency, an abnormal flow velocity profile in the ductus venosus and a tricuspid valve regurgitation represent indications for an echocardiographic examination. The presence of one or more of these markers is by far the most frequent indication for early echocardiography. Furthermore, this connection also offers the possibility to conduct a screening for heart defects as part of the first-trimester screening using these indirect markers, which are considerably easier and more common than the presentation of the four-chamber view, outflow tracts and major arteries. The potential of this approach has been examined in many investigations in recent years, particularly considering the still low detection rates for heart defects even in the second trimester [4]. A recently published meta-analysis of 20 studies, which were published between 1999 and 2012, covers 205232 fetuses with normal karyotype [41], 537 of which had a major heart defect. The analysis showed major differences in the respective detection rates of the individual studies, even for apparently similar study designs. Overall, the sensitivity and specificity of an NT above the 95th percentile (corresponding to an/NT of $2.5 \mathrm{~mm}$ ) were $44.4 \%$ (95\% CI: 39.5-49.5) and 94.5\% (95\% CI: 94.4-94.6), and, for an NT above the 99th percentile, 19.5\% (95\% CI: 15.9-23.5) and 99.1\% (95\% CI: 99.1-99.2) respectively; the results were similar when only studies in which the investigators were certified by the Fetal Medicine Foundation UK were considered; the sensitivity and specificity of an NT above the 95th percentile (corresponding to an NT of $2.5 \mathrm{~mm}$ ) were $45.6 \%$ (95\% CI: 39.6-51.7) and $94.7 \%$ (95\% CI: 94.6-94.9), and, for an NT above the 99th percentile, $21.0 \%$ (95\% CI: 16.5-26.1) and 99.2\% (95\% CI: 99.2-99.3) respectively [41].

Further, it was shown that, in the case of fetuses with thicker nuchal translucency, through the combination of thicker nuchal translucency and an abnormal flow pattern in the ductus veno- 
sus and/or the presence of tricuspid valve regurgitation, the detection rates for heart defects was increased for euploid fetuses. In a meta-analysis (9 studies of fetuses with thicker NT $[n=2908]$ and 7 studies of fetuses with normal NT [ $n=47610]$ ), in the case of thicker NT, the sensitivity of the abnormal ductus venosus flow pattern was $83 \%$, the specificity was $80 \%$, positive LH 4.4, negative LR 0.2 with a false positive rate of $20 \%$; in fetuses with normal NT, the sensitivity was $19 \%$, specificity $96 \%$, positive LH 5.0, negative LR 0.8 with a false positive rate of $4 \%$ [42].

A large study of 44456 euploid fetuses with 85 major heart defects showed that an NT in the $\geq 95$ th percentile would only have identified 30 (35\%) (false positive rate: $4.8 \%$ ) of heart defects, an NT in the $\geq 95$ th percentile or presence of a tricuspid valve regurgitation would have identified $43 \%$ (false positive rate: $5.9 \%$ ) and an NT in the $\geq 95$ th percentile or presence of a tricuspid valve regurgitation or an abnormal ductus venosus flow pattern would have identified $57.6 \%$ (false positive rate: $8.0 \%$ ) [37]. In a fixed false positive rate of $5 \%$, only $35 \%$ of heart defects would be detected through thicker NT (NT $\geq 95$ th percentile), $45 \%$ would have been detected as a result of NT and ductus venosus flow pattern, $49 \%$ through NT and tricuspid valve regurgitation and 54\% through NT and ductus venosus and tricuspid valve regurgitation [37].

A study from a centre in Barcelona showed similar results; here, over an eight-year period (2002-2009), 13773 single pregnancies with a crown-rump length of between $43 \mathrm{~mm}$ and $90 \mathrm{~mm}$, corresponding to the 11 th +0 to 14 th +4 weeks of pregnancy, as well as the blood flow pattern in the ductus venosus were measured [21]. The aim of the study was to use the ductus venosus blood flow not as a secondary marker in fetuses with thicker nuchal translucency as in other studies [42], but rather as a second marker alongside nuchal translucency thickness, as an earlier study of this group had shown that the additional evaluation of the ductus venosus blood flow profile can increase the sensitivity of the nuchal translucency thickness (NT >99th percentile) for heart defects in euploid fetuses by $11 \%$, from $29 \%$ to $40 \%$ [ 40 ]. In another group, $12.5 \%$ of euploid fetuses with normal nuchal translucency, in which a heart defect was later detected, showed an abnormal flow velocity profile in the ductus venosus, as, however, did $4.3 \%$ of healthy fetuses also [39]. 12799 normal fetuses were compared with the 37 euploid fetuses with major heart defects (in total, 760 fetuses had chromosomal or non-chromosomal anomalies). Of the 37 fetuses, $40 \%$ had an NT $>95$ th percentile, $27 \%$ had an NT $>99$ th percentile and $47 \%$ had an absent or reverse flow in the ductus venosus during atrial contraction. With the combination of an NT > 99th percentile and an abnormal flow profile in the ductus venosus (absent or reverse flow during atrial contraction) as a selection criterion, $47 \%$ of fetuses with heart defects and $2.7 \%$ of normal fetuses would have undergone a fetal echocardiogram [21].

It is certainly necessary for fetuses with the markers mentioned to undergo a fetal echocardiogram. However, the false positive rates for the respective markers are very high, and their positive predicted values are too low for them to be suitable for a general screening for heart defects. For the prenatal detection of heart defects, it appears more sensible and realistic to integrate the direct visualisation of the cardiac structures and cross-sections into the protocol of ultrasound screening examinations in the second semester, and further into the future, into the first-trimester screening as well.

\section{Early Echocardiogram as Part of the Detailed First-Trimester Screening $\nabla$}

It has been already shown that in experienced hands and with modern ultrasound equipment a majority of major $[8,13]$ and up to $65 \%$ of all heart defects [13] were able to be diagnosed by the end of the first trimester and beginning of the second trimester. A further analysis of the Lübeck data between 1992 and 2007 revealed that $66 / 77$ of heart defects (3521 fetuses examined) were detected between the 11 th +0 and 13 th +6 weeks of pregnancy, a further $9.1 \%$ in the second trimester, $2.6 \%$ in the third trimester and $2.6 \%$ postnatally [29]. Nowadays, a comprehensive echocardiogram is increasingly being carried out as part of sonographic examination of all fetal organs at prenatal medical centres, not only in the case of relevant risk (genetic, markers in first-trimester screening), but for all pregnant women as part of a detailed first-trimester screening [1,2,9]. Becker et al. [1] recently showed that the majority of severe, non-chromosomal anomalies $(72 / 94 ; 76.6 \%)$ arose in fetuses with "normal" NT and this could be diagnosed prenatally by means of a sonogram through the combination of a detailed first- and second-trimester screening, respectively in combination with an echocardiogram. The detection rates for severe anomalies during the firsttrimester screening were 58.6\% (65/111 cases) in the group with "normal" NT, 62.2\% (23/37) in the group with chromosomal anomalies and 56.8\% (42/74) in the group with non-chromosomal anomalies. Up to the $24 \mathrm{th}+0$ week of pregnancy, a further 24 anomalies were discovered in the group with "normal" NT, 9 of which were aneuploidies. With "normal" NT, the detection rate by the 24 th +0 week of pregnancy was $80.2 \%$ (89/111) and $87.4 \%$ (97/111) by birth. In 14 cases, aneuploidies (5 cases) and severe malformations ( 9 cases) were only diagnosed postnatally [1]. The authors requested that all pregnant women, regardless of the thickness of the fetal nuchal translucency, should be offered these examinations, and justified this in terms of ethics [1]. In a recently published study conducted at two large centres in Romania and Greece, 5472 fetuses were examined by a few very experienced examiners over 27 consecutive months (between January 2010 and March 2012) between the $12+0$ and $13+6$ weeks of pregnancy as part of an advance aneuploidy screening, a detailed organ diagnostic test including early echocardiography with appropriate, modern ultrasound machines [2]. A strict investigation protocol was executed for this. The duration of the examinations was between 18 and 52 minutes (average: 34 minutes), with between 24 and 62 images (average: 36 images) being saved per examination. In the case of an incomplete depiction of the fetal organs or cross-sections, the examination was carried out after a short break and/or a few days later; an additional transvaginal sonogram was necessary for 480 (7.80\%) of fetuses, either as a result of an unfavourable fetal position (5.59\%) or due to maternal factors, such as adiposity, myoma, abdominal scars or retroverted uterus $(2.21 \%)$. The prevalence of fetal anomalies was $3.0 \%$ (165/5472 cases). The detection rate for all non-chromosomal fetal anomalies was $40.6 \%$ (67/165 cases). The anomalies were divided in accordance with the RCOG's definition: “major (lethal or severe) anomalies: incompatible with life or associated with possible survival but severe immediate or long-term morbidity; moderate and minor anomalies: short or long-term morbidity of minor or moderate severity". The prevalence of major (lethal and severe) anomalies amounted to $1.39 \%$ (76/5472). $76 \%(58 / 76)$ of these were discovered at the first-trimester screening, i.e. $1.06 \%$ (58/5472) of the fetuses examined. An addi- 


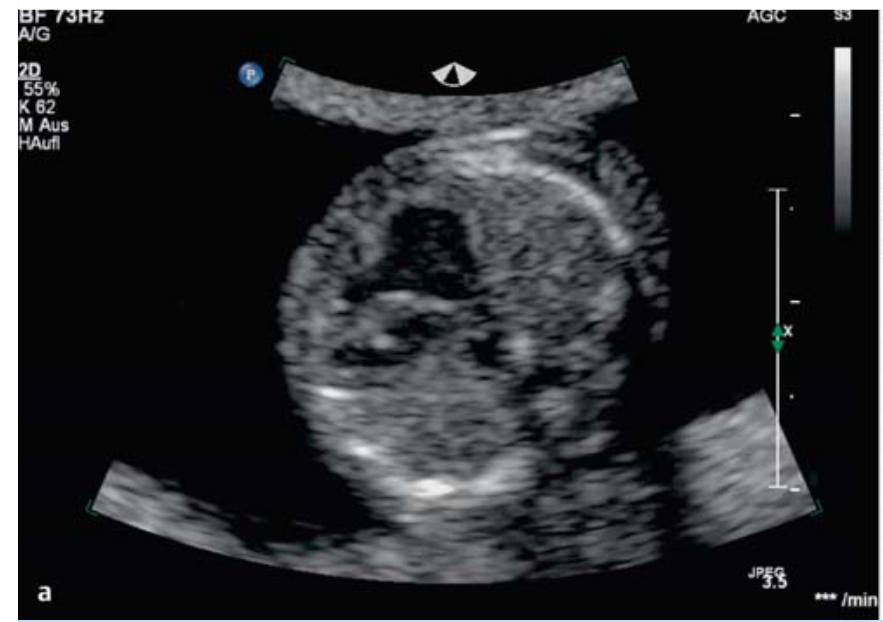

Fig. $\mathbf{1} \mathbf{a}$ and $\mathbf{b}$ Ebstein's anomaly with severe tricuspid valve insufficiency in a fetus at $13+0$ weeks and a crown-rump length of $70 \mathrm{~mm}$. a The already dilated right atrium of the heart is visible in the four-chamber view and, due to the apical approach of the tricuspid valve, has a very small right ventricle

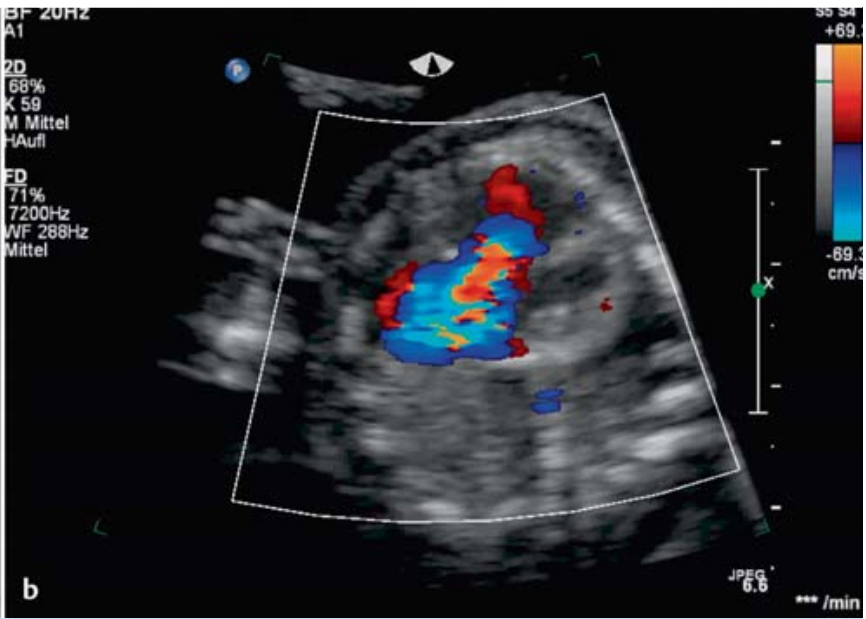

and/or larger atrial section of the right ventricle. $\mathbf{b}$ The transvaginal colour Doppler echocardiogram shows the severe tricuspid valve insufficiency, the jet of which begins apically and fills the right atrium. tional 16/5109 (0.31\%) had severe anomalies which were detected during the next ultrasound in the second trimester, with these being diagnosed postnatally in 2/4744 (0.04\%) fetuses. Minor/moderate anomalies were rarely detected in the early screening (10.1\%; 9/89), whereas 71 of the remaining 80 (79.8\%; $71 / 89$ ) were detected in the following examination in the second trimester; 9 of the anomalies in this group were only diagnosed postnatally. A thicker nuchal translucency (NT $>95$ th percentile) was exhibited in 25/76 (32.89\%) of fetuses with severe anomalies; $55.17 \%$ (16/29 fetuses) of fetuses with severe heart defects had a thicker cervix (5/12 [41.66\%] with isolated CHD; 11/17 with major CHD and extracardiac anomalies); 4 more were detected early as mild/moderate CHD. In total, the examiner can achieve a very high detection rate for severe heart defects in as early as the first-trimester screening, the prevalence of which was $0.54 \%$ (30/5472 fetuses), specifically 90\% (27/30 fetuses) [2]. A working group from Bari published two studies in which they evaluated the potential of early fetal echocardiography (11th to 14 th week of pregnancy) carried out by obstetric prenatal diagnostic experts for a high-risk group [17] and a low-risk group [9] with regard to the presence of a fetal heart defect. The period of study lasted six years (2005-2010). The abdominal situs, fourchamber view, right and left outflow tract, crossing over of the great arteries and three-vessel tracheal view were depicted by means of a two-dimensional and colour Doppler echocardiogram. A second echocardiographic examination was conducted by a fetal cardiologist between the 18th and 22nd weeks of pregnancy in the event of abnormalities. In the high-risk group (thickened NT in $73 \%$ of the fetuses) 802 of 870 fetuses displayed no abnormalities in both examinations [17], 36 had the same pathological findings in both examinations, and 32 were discordant. 6 had false positive findings in the early echocardiogram, 26 had other diagnoses, whereby the diagnosis was only slightly different or there were only slight anomalies in 14 cases (ventricular septal defect, mitral cleft, arteria lusoria). A severe heart defect was overlooked during the early echocardiogram in 6 out of 12 fetuses and, for a further 6 fetuses with anomalies detected in the early echocardiogram, the cardiac changes developed into a severe heart defect between the first and second examinations; the most common defect was outflow tract obstructions [17]. In the low-risk group, 4445 fetuses were examined and 42 heart defects were discovered (39 prenatal and 3 postnatal). In 27 cases, the fetal cardiologist confirmed the diagnosis, but in one case his diagnosis was slightly different and in 3 of the 26 cases which had been detected in the early echocardiogram, the heart defects developed progressively [9]. In both studies, the authors came to the conclusion that the early fetal echocardiogram is feasible and that most severe heart defects can be diagnosed by examiners well-versed in prenatal diagnostics; the appearance of some heart defects can change progressively until the second trimester, particularly semilunar valve stenosis and coarctatio aortae $[9,17]$.

\section{Features of Heart Defects to be Diagnosed in the First Trimester \\ $\nabla$}

The small size of the fetal heart in the first trimester limits the detectability of smaller, cardiac structural defects despite the use of higher frequency ultrasound, as a result of the relatively poorer detail resolution in the two-dimensional image in particular, but also in the colour Doppler image in comparison with the second-trimester screening. This is particularly the case for the frequent, small ventricular septal defects in the trabecular and outlet part of the interventricular septum; the interventricular shunts for these defects, which lead to their discovery in the second trimester $[43,44]$, are not currently detectable using colour Doppler. Other heart defects change cardiac structures as the pregnancy progresses further, meaning that they are detectable in the four- chamber view; this is particularly true for aortic and pulmonary valve obstructions, which are present in the first trimester and can also be detected through colour Doppler, but which could lead to abnormalities in the ventricle (myocardial hypertrophy, dilatation, hypoplasia, endocardial fibroelastosis) as the pregnancy progresses, depending on the severity of the obstruction $[5,8,45]$. The changes are similar in those leading to a narrow left ventricle and right heart dominance in the event of a severe obstruction in the first trimester in the context of aortic coarctation, with a normal four-chamber view potentially being seen until birth in the case of light obstruction [46]. Changes in 


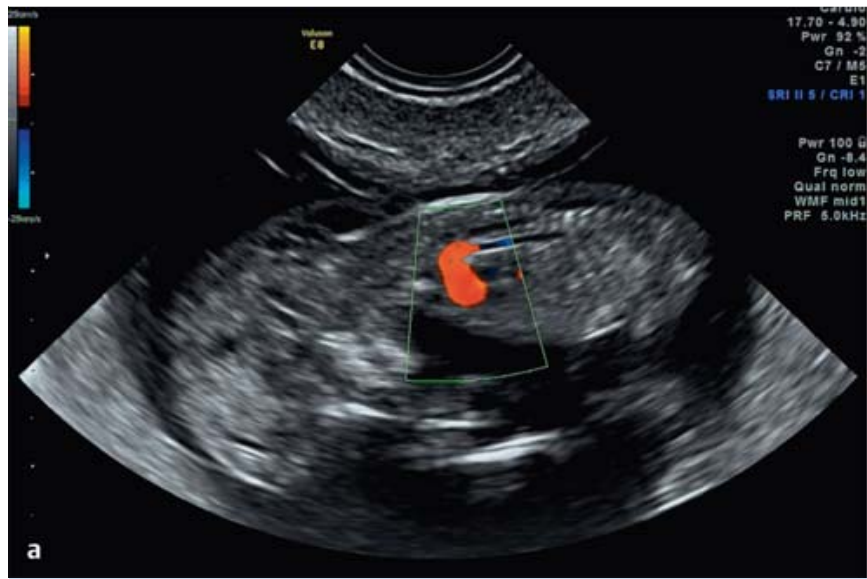

Fig. $\mathbf{2} \mathbf{a}$ and $\mathbf{b}$ Tetralogy of Fallot with absent pulmonary valve syndrome in a fetus with a crown-rump length of $63 \mathrm{~mm}$ at $12+3$ weeks of gestation. The karyotyping resulted in a trisomy $13(47, \mathrm{XY},+13)$. a The short axis shows a

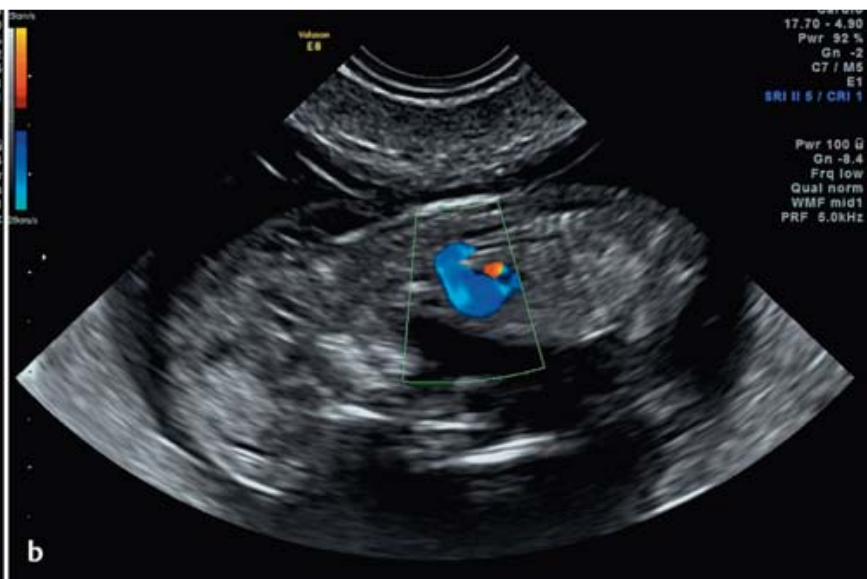

systolic forward flow into the truncus pulmonalis and into the ductus arteriosus, and $\mathbf{b}$ during diastole a backflow of the descending aorta via ductus arteriosus and truncus pulmonalis in both heart chambers.

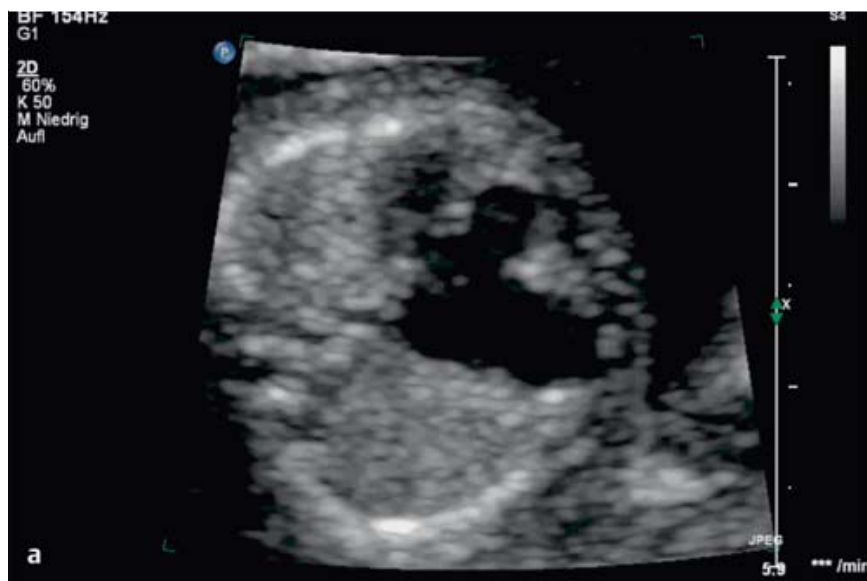

Fig. $\mathbf{3}$ a and b Complete atrioventricular septal defect in a fetus with a crown-rump length of $82 \mathrm{~mm}$ at $12+2$ weeks of gestation. a In the fourchamber view, the common atrioventricular valve, the ventricular septal de-

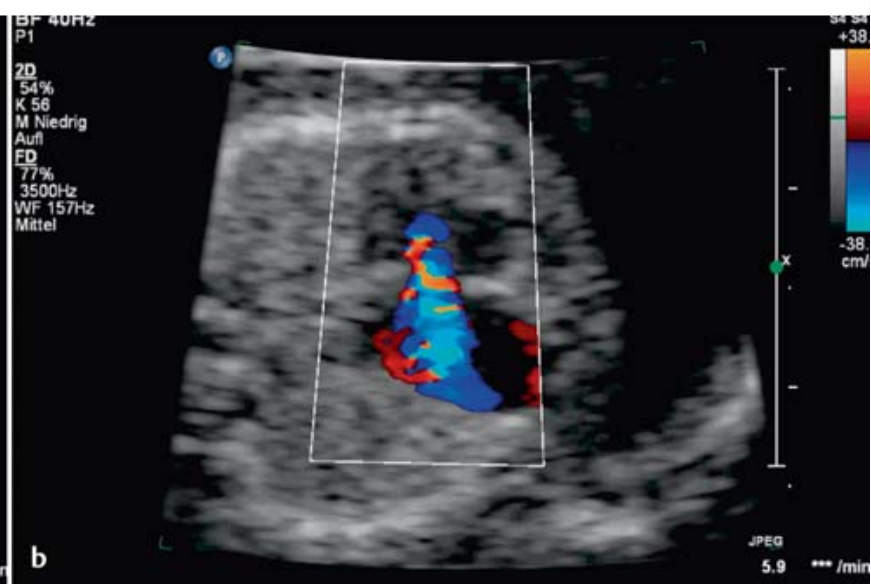

fect underneath and the defect of the septum primum in the atrial area directly above the valve can be detected. b The colour Doppler echocardiogram shows a severe insufficiency of the common atrioventricular valve. an Ebstein's anomaly are also frequently not manifested until the second trimester, although the frequently associated severe tricuspid valve insufficiency can also be detected in as early as the first trimester [47]. However, fetuses with very severe tricuspid valve insufficiencies in the case of Ebstein's anomaly ( $\bullet$ Fig. 1 a and b) and tricuspid valve dysplasia, discovered in the first trimester, can die in utero. It is particularly common for fetuses to die quickly after the first trimester with a tetralogy of Fallot with absent pulmonary valve syndrome and open ductus arteriosus ( Fig. 2 a and $\mathbf{b}$ ), as the massive backflow due to pulmonary insufficiency in the event of an existing ventricular septal defect leads to an enormous overload in both ventricles [48]. The progress of severe stenosis affecting both semilunar valves can be similar. More frequently depicted and ultimately to be diagnosed in the first and the second trimester are atrioventricular septal defect ( Fig. $3 \mathbf{a}$ and b), tricuspid valve atresia, major ventricular septal defect, pulmonary and aortic atresia on the basis of the absence of the visualisation of one of the two major arteries and their hypoplasia with reverse flow in the colour Doppler, the hypoplastic left heart in the case of a pulmonary atresia with intact ventricular septum and malpositioning of the major arteries, such as double outlet ventricle and transposition of the major arteries ( Fig. 4), truncus arteriosus communis and the tetralogy of Fallot ( Fig. 5), although here the overriding of the aorta and their increase in size in the case of smaller ventricular septal defects is not as clear and easy to detect in the first trimester as in the second [16]. Even more complex anomalies occurring in heterotaxy syndrome with vena azygos continuity of the inferior vena cava can be detected, as can the presence of a left persistent superior vena cava and of anomalies in the aortic arch, such as a right aortic arch and an arteria lusoria [49]. Cardiomyopathies and heart tumours normally first appear in the second half of pregnancy [8].

\section{Conclusion for Clinical Practice \\ $\nabla$}

The majority of major heart defects can be diagnosed through a detailed examination of the fetal heart as part of a fetal echocardiogram in the 12 th and 13 th weeks of pregnancy. A re- 


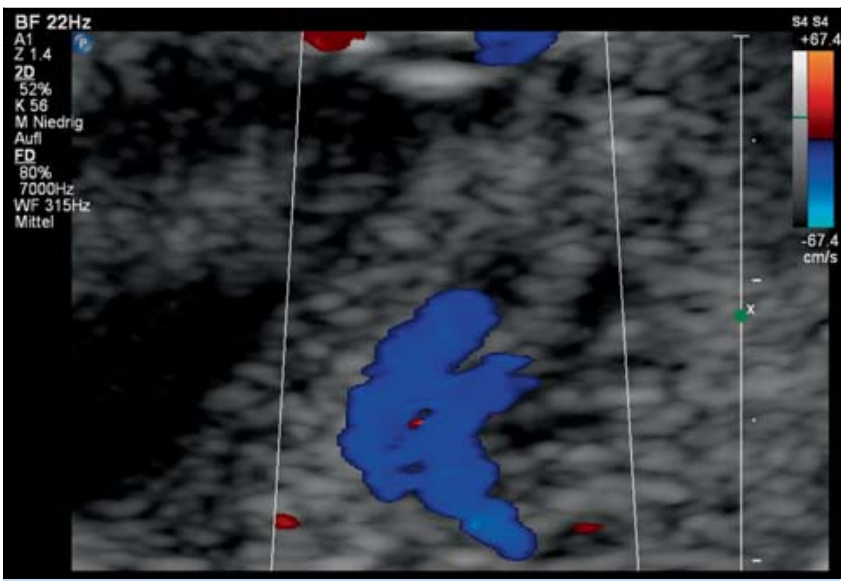

Fig. 4 Transposition of the major arteries in a fetus with a crown-rump length of $86 \mathrm{~mm}$ at $13+5$ weeks of gestation. The parallel course of both great arteries with the ascending aorta to the front and right is easy to recognise in the colour Doppler.

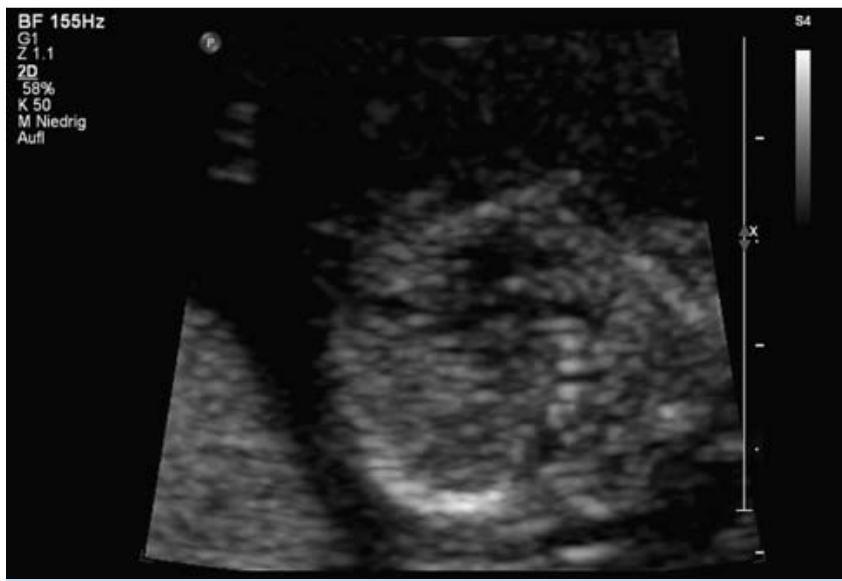

Fig. 5 Tetralogy of Fallot in a fetus with a crown-rump length of $65 \mathrm{~mm}$ at $12+6$ weeks of gestation. Showing the left ventricular outflow tract, a ventricular septal defect and a broad overriding and right ascending aorta were detected. quirement for this is the segmental approach with the visualisation of all connections and the required cross-sections, as well as the use of the transvaginal echocardiogram in the case of inadequate visualisation, and the repetition of the examination after a short time interval if applicable. Besides anamnestic risk, a thickened nuchal translucency, the increased pulsatility of the blood flow pattern in the ductus venosus as well as the presence of a tricuspid valve regurgitation are significant markers for the presence of a heart defect.

\section{Conflict of Interest}

None.

\section{References}

1 Becker R, Schmitz L, Kilavuz S et al. "Normal" nuchal translucency: a justification to refrain from detailed scan? Analysis of 6858 cases with special reference to ethical aspects. Prenat Diagn 2012; 32: 550-556

2 Iliescu D, Tudorache S, Comanescu A et al. Improved detection rate of structural abnormalities in the first trimester using an extended examination protocol. Ultrasound Obstet Gynecol 2013; 42: 300-309

3 Becker R, Wegner RD. Detailed screening for fetal anomalies and cardiac defects at the 11-13-week scan. Ultrasound Obstet Gynecol 2006; 27: 613-618

4 Gembruch U, Geipel A. Indication for fetal Echocardiography: Screening in low and high Risk Population. In: Yagel S, Silverman NH, Gembruch U, eds. Fetal Cardiology. 2nd ed. New York: Informa Healthcare; 2009: 111-129

5 Gembruch U, Geipel A, Herberg $U$ et al. Kardiale Interventionen bei Feten. Z Geburtsh Neonatol 2012; 216: 162-172

6 Gembruch U, Knöpfle G, Chatterjee $M$ et al. First-trimester diagnosis of fetal congenital heart disease by transvaginal two-dimensional and Doppler echocardiography. Obstet Gynecol 1990; 75: 496-498

7 Gembruch U, Knöpfle G, Bald R et al. Early diagnosis of fetal congenital heart disease by transvaginal echocardiography. Ultrasound Obstet Gynecol 1993; 3: 310-317

8 Yagel S, Weissman A, Rotstein Z et al. Congenital heart defects: natural course and in utero development. Circulation 1997; 96: 550-555

9 Volpe $P$, Ubaldo P, Volpe $N$ et al. Fetal cardiac evaluation at 11-14 weeks by experienced obstetricians in a low-risk population. Prenat Diagn 2011; 31: 1054-1061

10 Axt-Fliedner R, Chiriac A, Gembruch U. First and early second trimester fetal heart scanning. Ultraschall Med 2009; 30: 364-375
11 Khalil A, Nicolaides $\mathrm{KH}$. Fetal heart defects: potential and pitfalls of first-trimester detection. Semin Fetal Neonat Med 2013; 18: 251-260

12 Smrcek JM, Berg C, Geipel A et al. Early fetal echocardiography: heart biometry and visualization of cardiac structures between 10 and 15 weeks' gestation. J Ultrasound Med 2006; 25: 173-182

13 Smrcek JM, Berg C, Geipel A et al. Detection rate of early fetal echocardiography and in utero development of congenital heart defects. J Ultrasound Med 2006; 25: 187-196

14 Vimpelli T, Huhtala H, Acharya G. Fetal echocardiography during routine first-trimester screening: a feasibility study in an unselected population. Prenat Diagn 2006; 26: 475-482

15 Geipel A, Gembruch U. Fetale Nackentransparenzmessung und weitere Ersttrimester Marker: Screening auf fetale Herzfehler. Z Geburtsh Neonatol 2012; 216: 157-161

16 Bhat AH, Kehl DW, Tacy TA et al. Diagnosis of tetralogy of Fallot and its variants in the late first and early second trimester: details of initial assessment and comparison with later fetal diagnosis. Echocardiography 2013; 30: 81-87

17 Volpe P, De Robertis V, Campobasso G et al. Diagnosis of congenital heart disease by early and second-trimester fetal echocardiography. Ultrasound Med 2012; 31: 563-568

18 Persico N, Moratalla J, Lombardi CM et al. Fetal echocardiography at 1113 weeks by transabdominal high-frequency ultrasound. Ultrasound Obstet Gynecol 2011; 37: 296-301

19 Moon-Grady A, Shahanavaz S, Brook M et al. Can a complete fetal echocardiogram be performed at 12 to 16 weeks' gestation? J Am Soc Echocardiogr 2012; 25: 1342-1352

20 Allan L, Benacerraf B, Copel JA et al. Isolated major congenital heart disease. Ultrasound Obstet Gynecol 2001; 17: 370-379

21 Borrell A, Grande M, Bennasar M et al. First-trimester detection of major cardiac defects with the use of ductus venosus blood flow. Ultrasound Obstet Gynecol 2013; 42: 51-57

22 Westin M, Saltvedt S, Bergman G et al. Routine ultrasound examination at 12 or 18 gestational weeks for prenatal detection of major congenital heart malformations? A randomized controlled trial comprising 36,299 fetuses. BJOG 2006; 113: 675-682

23 Syngelaki A, Chelemen T, Dagklis T et al. Challenges in the diagnosis of fetal non-chromosomal abnormalities at 11-13 weeks. Prenat Diagn 2011; 31: 90-102

24 Gembruch U. Prenatal diagnosis of congenital heart disease. Prenat Diagn 1997; 17: 1283-1298

25 Yagel S, Arbel R, Anteby EY et al. The three vessels and trachea view (3VT) in fetal cardiac scanning. Ultrasound Obstet Gynecol 2002; 20: 340-345

26 Lee W, Allan L, Carvalho JS et al.; ISUOG Fetal Echocardiography Task Force. ISUOG consensus statement: what constitutes a fetal echocardiogram? Ultrasound Obstet Gynecol 2008; 32: 239-242 
27 Chaoui R, Heling K, Mielke G et al. Qualitätsanforderungen der DEGUM zur Durchführung der fetalen Echokardiografie. Ultraschall Med 2008; 29: $197-200$

28 Gembruch U, Shi C, Smrcek JM. Biometry of the fetal heart between 10 and 17 weeks of gestation. Fetal Diagn Ther 2000; 15: 20-31

29 Hartge DR, Weichert J, Krapp $M$ et al. Results of early foetal echocardiography and cumulative detection rate of congenital heart disease. Cardiol Young 2011; 21: 505-517

30 Votino C, Cos T, Abu-Rustum R et al. Use of spatiotemporal image correlation at 11-14 weeks' gestation. Ultrasound Obstet Gynecol 2013; 42: 669-678

31 Bronshtein M, Zimmer EZ. The sonographic approach to the detection of fetal cardiac anomalies in early pregnancy. Ultrasound Obstet Gynecol 2002; 19: 360-365

32 Clur SA, Ottenkamp J, Bilardo CM. The nuchal translucency and the fetal heart: a literature review. Prenat Diagn 2009; 29: 739-748

33 Makrydimas G, Sotiriadis A, Huggon JC et al. Nuchal translucency and fetal cardiac defects: A pooled analysis of major fetal echocardiography centers. Am J Obstet Gynecol 2005; 192: 89-95

34 Berg C, Kremer C, Geipel A et al. Ductus venosus blood flow alterations in fetuses with obstructive lesions of the right heart. Ultrasound Obstet Gynecol 2006; 28: 137-142

35 Maiz N, Plasencia W, Dagklis T et al. Ductus venosus Doppler in fetuses with cardiac defects and increased nuchal translucency thickness. Ultrasound Obstet Gynecol 2008; 31: 256-260

36 Faiola S, Tsoi E, Huggon IC et al. Likelihood ratio for trisomy 21 in fetuses with tricuspid regurgitation at the 11 to $13+6$-week scan. Ultrasound Obstet Gynecol 2005; 26: 22-27

37 Pereira S, Ganapathy R, Syngelaki A et al. Contribution of fetal tricuspid regurgitation in first-trimester screening for major cardiac defects. Obstet Gynecol 2011; 117: 1384-1391

38 Chelemen T, Syngelaki A, Maiz $N$ et al. Contribution of ductus venosus Doppler in first-trimester screening for major cardiac defects. Fetal Diagn Ther 2011; 29: 127-134

39 Prats $P$, Ferrer $Q$ Comas $C$ et al. Is the addition of the ductus venosus useful when screening for aneuploidy and congenital heart disease in fetuses with normal nuchal translucency? Fetal Diagn Ther 2012; 32: $138-143$
40 Martínez JM, Comas M, Borrell A et al. Abnormal first-trimester ductus venosus blood flow: a marker of cardiac defects in fetuses with normal karyotype and nuchal transluceny. Ultrasound Obstet Gynecol 2010; 35: $267-272$

41 Sotiriadis A, Papatheodorou S, Eleftheriades $M$ et al. Nuchal translucency and major congenital heart defects in fetuses with normal karyotype: a meta-analysis. Ultrasound Obstet Gynecol 2013; 42: 383389

42 Papatheodorou SI, Evangelou E, Makrydimas G et al. First-trimester ductus venosus screening for cardiac defects: a meta-analysis. BJOG 2011; 118: $1438-1445$

43 Axt-Fliedner R, Schwarze A, Smrcek J et al. Isolated ventricular septal defects detected by color Doppler imaging: evolution during fetal and first year of postnatal life. Ultrasound Obstet Gynecol 2006; 27: 266273

44 Gómez O, Martínez J, Olivella A et al. Isolated ventricular septal defects in the era of advanced fetal echocardiography: risk of chromosomal anomalies and spontaneous closure rate from diagnosis to the first year of life. Ultrasound Obstet Gynecol 2013; DOI: 10.1002/uog.12527

45 Axt-Fliedner R, Kreiselmaier P, Schwarze A et al. Development of hypoplastic left heart syndrome after diagnosis of aortic stenosis in the first trimester by early echocardiography. Ultrasound Obstet Gynecol 2006; 28: $106-109$

46 Axt-Fliedner R, Hartge D, Krapp M et al. Course and outcome of fetuses suspected of having coarctation of the aorta during gestation. Ultraschall Med 2009; 30: 269-276

47 Gembruch U, Kohl T, Geipel A et al. Ebstein-Anomalie des Feten. Gynäkologe 2006; 39: 39-47

48 Berg C, Thomsen Y, Geipel A et al. Reversed end-diastolic flow in the umbilical artery at $10-14$ weeks of gestation is associated with absent pulmonary valve syndrome. Ultrasound Obstet Gynecol 2007; 30: 254258

49 Rembouskos G, Passamonti U, De Robertis Vet al. Aberrant right subclavian artery (ARSA) in unselected population at first and second trimester ultrasonography. Prenat Diagn 2012; 32: 968-975 